\title{
Le contrat et le sacrifice dans la poétique d'Aubigné
}

\author{
GISÈLE MATHIEU-CASTELLANI
}

Du Printemps aux Tragiques, nulle solution de continuité ne rompt la cohérence de l'œuvre d'Aubigné: non seulement les groupements thématiques, le système des images et des figures, les réseaux signifiants dessinant son mythe personnel y demeurent stables, à travers la différence des registres discursifs, mais encore une même activité s'y déploie, de pro-testation; de témoignage devant un Tribunal imaginaire, appelé à voir et à juger la culpabilité et l'innocence, à donner au crime exemplaire un châtiment exemplaire.

Cette insistance d'un même schème dynamique qui fait monter la protestation vers le Ciel se remarque jusque dans les coïncidences de certains montages structurels: le dernier sonnet de L'Hécatombe, clôturant la centaine en lui assurant l'unité d'une petite totalité, ${ }^{1}$ le dernier livre des Tragiques, septième sceau fermant l'œuvre dans sa perfection symbolique, mettent également en scène un procès tenu devant une Cour de Justice, "la celeste Cour / Où se preuvent les mains innocentes ou pures" (Héc. C, v. 56), le Tribunal céleste où, en "cet acte dernier" (VII, v. 753) de la Tragédie, L'Éternel vient juger, prononçant "la dernière sentence" (v. 864).

Dans Le Printemps, dans Les Tragiques, la mise en scène judiciaire éclaire une situation triangulaire opposant accusé, accusateur et juge, qui reproduit une situation dramatique fondamentale à trois personnages, bourreau, victime, témoins. Ici et là, cette situation se constitue en spectacle, sous l'oeil d'un Voyeur tout-puissant, haut placé, appelé à l'apprécier "car ceci du haut ciel tu verras" (V, v. 361).

* * *

Néanmoins, de l'un à l'autre texte, la situation communicationnelle est marquée par un dispositif différent. Dans Le Printemps, la relation initiale est duelle: conformément à la topique du Canzoniere, un locuteur s'adresse à un allocutaire dans un discours persuasif, écrit ici en code néopétrarquiste. Mais chacun des deux rôles principaux se dédouble: le locuteur y parle en tant que victime, liée à son bourreau par le jeu sado- 
masochiste, et en tant que témoin de l'accusation, s'adressant à un coupable qu'il s'agit de faire condamner. L'allocutaire de son côté s'y constitue à la fois en tant que destinataire d'un message amoureux, et en tant que juge-arbitre, appelé à apprécier les dommages subis:

Ma maistresse sera pour moy à ce besoin,

Je la veux pour arbitre, ou juge, ou pour tesmoin

(Héc. XLV, 107, v. 9-10)

En tant qu'accusateur, le locuteur veut contraindre le juge-arbitre-témoin à attester la véracité d'un discours inscrit dans les marques corporelles comme autant de lettres sanglantes:

J'ouvre mon estommac, une tumbe sanglante

De maux enseveliz: pour Dieu, tourne tes yeux,

Diane, et voy au fond mon cueur party en deux

Et mes poumons gravez d'une ardeur viollente...

(St. VI, 211),

à voir ce qu'il ne veut pas voir; en tant que victime, il entend établir un pacte avec son bourreau, et opérer, par l'auto-mutilation sacrificielle, une médiation qui détourne la violence. Faute de succès, à la médiation succède le recours au système judiciaire, oû se reconstitue la scène triangulaire tribunal / accusé / accusateur:

Au tribunal d'amour, apprès mon dernier jour,

Mon coeur sera porté, diffamé de bruslures,

Il sera exposé...

(C 168, v. 1)

Dans Les Tragiques, le rôle du destinataire est tenu par deux acteurs; l'interlocuteur privilégié est Dieu, qui a "donné l'argument" (L'Autheur à son Livre, v. 410), et qui est garant de la véridicité affichée:

Ainsi les visions qui seront ici peintes

Seront exemples vrais...

(VI, v. 89-90),

caution d'autant plus irrécusable que l'organe humain n'est que l'instrument de la parole divine:

Que je ne sois qu'organe à la celeste voix

(VI, v. 59),

et que tout mensonge est interdit lorsque l'allocutaire voit tout: "mentir je ne t'ose" (IV, v. 37, discours à la Conscience). Les intẹrlocuteurs secondaires 
sont les hommes, divisés en deux classes, les enfants de vérité, les serfs de vanité, un auditoire constitué soit en témoins, soit en accusés. Si le premier allocutaire atteste la véridicité de la narration, les autres justifient la mise en discours / mise en récit de la vérité. ${ }^{2}$

À ce dédoublement du destinataire, correspond un double jeu du destinateur; en tant qu'organe de la céleste voix, s'adressant à son allocutaire privilégié, Dieu, dont il s'autorise pour reproduire sa parole:

Il n'y a rien du mien ni de l'homme en ce lieu.

Voici les propres mots des organes de Dieu.

(VII, v. 221-2),

il a pour projet de le contraindre à juger, à punir / récompenser:

Porte l'heur ou malheur, l'arrest que je prononce

(VII, v. 10)

S'adressant, d'autre part, en tant que porte-parole des opprimés, aux hommes qu'il lui faut transformer en témoins de l'injustice, il joue le rôle de l'avocat, "Qui l'oreille et le coeur anime des François" (VI, v. 60):

Ames dessous l'autel victimes des idoles,

Je preste à vos courroux le fiel de mes paroles

(IV, v. 53-54),

intermédiaire entre victimes et juge.

Quel que soit le dispositif de la mise en scène judiciaire, une constante se fait jour: c'est en terme d'obligation réciproque que se définissent les relations de l'allocuteur à l'allocutaire; soumis à l'autorité d'une voix d'en haut qui édicte ses impératifs, l'allocuteur pourtant s'efforce de "posséder" son allocutaire, Maître ou Maîtresse, Dieu ou Diane, en le forçant d'abord à voir les scènes qu'il construit en régisseur du spectacle, comme autant de visions exemplaires, ensuite à jouer le rôle d'arbitre. Un contrat se met en place dans cette situation de communication particulière, avec distribution des rôles, et obligations de part et d'autre: du côté du locuteur, l'obligation de dire vrai, constamment affirmée comme un impératif; au lieu des "charmeresses feintes" un discours de la vérité adressé aux enfans de vérité (VI, 1106-7), au lieu d'une plainte mensongère, le discours d'un coeur et d'un corps souffrants: si "mesmes signes ont l'amour vraye et la feincte" (Héc. LIV, 117, v. 10), la mise à nu attestera la vérité d'une passion non feinte, en guise de "preuve d'amour" irrécusable (Héc. LIV, 117, v. 13-14). Du côté de l'allocutaire, l'obligation de tenir parole, de respecter sa promesse: "Comme tu as promis, donne..." (VI, v. 15).

Quel que soit le registre discursif, et le "genre d'escrire", un même type de 
contrat détermine les rapports entre partenaires: analogue à celui qui lie le Prince à ses sujets, un même réseau de "debvoirs mutuels" oblige le fidèle et son Dieu, le serviteur et sa maîtresse, sur le mode galant ou dramatique dans Le Printemps, sur le mode tragique dans Les Tragiques.

La situation de communication se trouve donc régie par ce réseau d'obligations réciproques qui, lorsqu'il s'actualise dans une mise en scène judiciaire, comme on l'a vu, est à mettre en rapport avec les structures rhétoriques des recueils, où le discours-cadre est précisément de type judiciaire. ${ }^{3}$ Mais le contrat qui fixe la répartition des rôles dans la situation de communication est aussi le reflet du contrat qui, dans l'imaginaire d'Aubigné, détermine les relations entre partenaires, dans la vie sociale, dans la vie amoureuse, dans la vie religieuse. L'insistance à établir un pacte, à fixer une convention, à distribuer dettes et obligations, témoigne d'une obsession du lien juridique, qui se révèle dans toute l'œuvre d'Aubigné, quel que soit son registre.

Il faut rappeler l'abondant lexique qui renvoie à l'intertexte juridique et judiciaire: contrats et conventions, conventions et promesses, traité, caution, bonne foy, foy jurée, foy violée, rompre la loy, octroy, dispositions, etc. dans le Deb voir mutuel; mais aussi dans Le Printemps, de façon plus étonnante, faire preuve, procès, cause, juge, loix, faire preuve, secours, assister (en justice), rançon, plaider, ajourner, demander la somme et l'interest, reparer l'injustice et l'injure, payerl'usure, arbitre, juge ou tesmoin, récuser, droipt escript ne coutume ne loix, juge et partie, bannir, condamné, tribunal, celeste Cour, preuve, demander justice au Juge, accuser, punir, peines, forfait, punition, justice, suplice, etc.; et jusque dans les Meditations sur les Pseaumes: termes et mauvais payeurs, loix et obligation, arrhe, gage (496), recognoissance et hypothèque, tiltre (de propriété), faire le procès (498), traictez, se soustraire aux charges, exercer la justice (499), tributs (503), succession (506), gages (513), reparer les fautes, payer la peine, salaires (514), requestes, payer la rançon, héritage (515), garantir, potences (516), prisons, sentence, asile, exil (520), advocat (537), justice, criminels, cachots, forfait (538), corrompre le juge, forfaiture (539), etc. Ce lexique est particulièrement important, on pouvait s'y attendre, dans Les Tragiques, notamment aux livres III Chambre Dorée, VI Vengeances, et VII Jugement, dont les titres mêmes évoquent le lieu, la finalité, et les rituels d'un même procès, toujours recommencé ...

Outre la présence insistante de ce lexique, la répétition obsédante de scènes identiques, aisément superposables dans toute l'œuvre, où se tient un procès devant une cour, où se plaide une défense, ou un réquisitoire, où se prononce un jugement, et s'exécute une sentence, comme, parallèlement, la transformation du locuteur en avocat ou en procureur, et de 
l'allocutaire en témoin, en arbitre ou en juge, sont l'indice de la fascination qu'exerce sur l'imaginaire d'Aubigné le rituel de la Justice et de ses œuvres. Rappelons seulement cette scène dramatique, rapportée dans $S a$ vie à ses enfants: le jeune Agrippa, âgé de huit ans et demi, passe par Amboise en compagnie de son père, y voit "les testes de ses compagnons d'Amboise encore recognoissables sur un bout de potence", et se voit imposer par la voix paternelle, de façon solennelle, l'alternative, vengeance ou malédiction .... ${ }^{4}$

Parmi l'ensemble des activités de la vie judiciaire, Aubigné privilégie, dans les contrats et les conventions, les traités et les pactes, tout ce qui garantit ou doit garantir le respect de la parole lorsqu'elle s'engage dans une promesse qui vaut pour un acte, lorsque dire, c'est faire, tout ce qui règle les modalités de l'échange, du don et du contre-don, de la dette et de la créance. La notion juridique de contrat, qui fonde la loi morale, est au centre de la vision du monde d'Aubigné: elle règle à la fois et de la même manière, outre le rapport du fils au père dans la fiction autobiographique, les relations sociales au sein de la Cité, les relations sentimentales et érotiques dans le commerce amoureux, les relations du croyant à Dieu dans l'échange spirituel.

Du côté du politique, le titre du traité Du Debvoir mutuel Des Roys et des Subects rappelle un passage des Vindiciae: "on voit assez quel est et quel doit estre le droit et le debvoir du prince envers le peuple et du peuple envers le prince, et que ces debvoirs mutuels sont distinguez l'un de l'autre". ${ }^{5}$ Mais en dépit du titre, le texte d'Aubigné met l'accent sur le devoir du Prince, plus que sur ses droits, sur les droits des sujets, plus que sur leur devoir, alors que le Vindiciae, dont le titre français est "De la puissance légitime du Prince sur le peuple, et du peuple sur le Prince", examine les conditions qui autorisent le droit à la résistance. Déplacement de perspective: Aubigné y révèle son souci obsédant de lier le Prince, de l'ob-liger, et en particulier, de le contraindre à tenir ses engagements:

Voicy ce que vous avez mis en question:

Si les traittez, contracts et conventions entre le Prince et ses subjects sont obligatoires de la part du Prince.

Par quels moyens legitimes le Prince peut estre adstrainct à l'observation des conventions et promesses faites à ses subjects.

Quelles cautions et asseurances le peuple peut demander à son Prince pour l'observation des conventions et promesses. ${ }^{6}$

Le motif de la promesse y détermine les motifs associés par contraste de la fidélité: "nous voulons examiner si ces Roys (...) doivent souffrir quelques loys, et si de ces loys ils doibvent estre protecteurs ou destructeurs, s'ils ont quelques regles ou s'il faut qu'ils soyent desreglez, s'ils doivent garder quelque loyauté ou estre infideles et desloyaux;"7, et de la foy violée qui 
exige réparation et vengeance. 8 "Le Prince qui rompt la foy à son peuple rompt celle de son peuple":9 ici encore la thématique du contrat et de la promesse qui le scelle rencontre la règle de l'obligation, comme, ailleurs, dans le registre satirique: "Tout Prince qui voudra régner sans qu'on le barbouille d'équité et sans estre controllé de la parole de Dieu, il faut qu'il extermine les Huguenots." (Confession du Sieur de Sancy, p. 649). Notons en outre que dans le traité du Debvoir Mutuel se déclare une même obsession de pro-tester, avec la même image du sang qui témoigne, et monte pour se donner en spectacle au monde et au ciel, pour exiger récompense: "soyent le Ciel et le Monde spectateurs du sang que nous espandons...."10

Du côté des Amours, la même thématique du contrat et de la promesse soutient les imprécations contre celles "qui ont foullé au pied les promesses jurées" (Stances, IV, v. 71), et une même obsession de la règle d'équivalence dans l'échange, donnant-donnant, fait exiger "sang pour sang et vie pour la vie" (Stances IV, v. 102) tandis que le même souci de l'exact salaire conduit à déclarer la perversité de l'échange amoureux, là où est transgressée la loi d'équivalence.

Du côté du lyrisme religieux, enfin, se manifeste une même volonté de lier le fidèle et son Dieu par un contrat engageant l'un et l'autre dans un réseau de debvoirs mutuels, donnant-donnant:

Elle crie en son lict: "Ô dieu double ma foy,

Je ne t'oublieray point, mais, mon Dieu fay en sorte

Qu'à la force du mal je devienne plus forte

(IV, v. 1031-4)

Je ne t’ay point laissé, ne m’abandonne pas,

$\mathrm{Ne}$ me laisse. Seigneur, de peur que je te laisse

(IV, v. 600-602)

Un même rappel de la promesse:

Comme tu as promis, donne...

(VI, v. 15)

Et un même désir de forcer Dieu à respecter la règle de l'équivalence, espérée de sa Justice:

Voyez quels justes poids quelles justes balances

Balancent dans les mains des celestes vengeances

(VI, v. 905-906)

Dans cette insistance remarquable des thèmes de la convention et de la promesse, quelque soit le type de discours, le motif du sacrifice, dont la 
récurrence est notable, apparaît comme l'une des modalités possibles du contrat qui lie deux parties: "Il n'y a pas de sacrifice, comme dit M. Mauss, qui n'ait quelque chose de contractuel."11 Dans Le Printemps, les cent sonnets de L'Hécatombe inscrivent dans leur titre même le thème sacrificiel; et ils sont présentés comme l'exact équivalent des cent taureaux immolés à Diane-Artémis pour apaiser son courroux. Les Stances (notamment VI et VIII) mettent en scène le geste spectaculaire de l'ouverture sanglante d'un estomac devenu autel sur lequel est sacrifié le corps d'une victime innocente. Dans Les Tragiques, les livres IV et V en particulier ont pour objet le sacrifice librement consenti des victimes candides (IV, v. 14), "Ames dessous l'autel victimes des idoles" (IV,v. 52), et leur martyre fait de leurs bourreaux des Caïns (V, v. 1550) répétant le premier sacrifice, le premier sang versé.

Dans Le Printemps, dans Les Tragiques, trois modalités du sacrifice ${ }^{12}$ s'associent selon la règle de l'équivalence ou du contraste: l'immolation, "qu'on fait par effusion de sang", l'holocauste, "quand l'hostie est consumée par le feu", et "le véritable sacrifice", l'offrande d'un coeur et d'un corps purs, geste par lequel "l'homme s'offre lui-même, pur, immaculé, en hostie vivante à dieu, à l'exemple du souverain prêtre le Christ."13

L'immolation. Aux sonnets XCVI et XCVII de L'Hécatombe, répond la séquence du livre VI des Tragiques (v. 177 sq.) consacrée à la narration du meurtre d'Abel. Ici et là, au corps de l'animal émissaire, intermédiaire entre le destinateur et le destinataire du sacrifice, se substitue, par une transgression, le corps d'une victime innocente. Dans une substitution seconde, le corps, dans le sonnet XCVI, est remplacé par le corpus, les cent sonnets qui en tiennent lieu. Mais de la même manière, dans les deux cas, le sacrifice est offert au courroux de la divinité, ou de celui qui usurpe son rôle:

A moins de cent taureaux on ne fait cesser l'ire

De Diane en courroux

(Héc. XCVI, v. 6-7)

Il massacra son frere et de cet agneau doux

Il fit un sacrifice à son amer courroux

(VI, v. 183-184)

Sacrifice pervers, subvertissant les rites et les règles de l'échange, mais qui lie la victime à son bourreau, le bourreau à sa victime. Le sang versé oblige celui pour qui il a coulé.

L'holocauste. Les sonnets XCVI et XCVII, comme les Stances VI, confondent, dans un même acte sacrificiel marqué par l'ouverture de l'estomac exhibant ses parties, immolation et holocauste. 
Je brusle avec mon ame et mon sang rougissant

Mais quoy puis je cognoistre au creux de mes hosties

A leurs boyaux fumans, à leurs rouges parties

Ou l'ire, ou la pitié de ma divinité?

(XCVI)

Ouy je suis proprement à ton nom immortel

Le temple consacré, tel qu'en Tauroscythie

Fust celuy où le sang appaisoit ton envie,

Mon esthomac pourpré est un pareil autel.

....

L'holocoste est mon cœur...

(XCVII)

Voy mon sang escumeux...

.

Tu me brusle...

La combinaison des deux modes du sacrifice joue des deux registres symboliques: le sang y témoigne de façon irrécusable, comme dans toute l'œuvre d'Aubigné, de la violence insupportable, il marque le martyre en le signalant à l'œil comme preuve; le feu, à la fois destructeur et régénérateur, produit des cendres, promesse de vie.

De même, dans Les Tragiques, les victimes dont le sang est versé deviennent martyrs, témoins irrécusables du désordre et de l'injustice, tandis que les bûchers sur lesquels sont sacrifiés les opprimés transforment les cendres des bruslés en précieuses graines.

L'offrande. Les deux premières formes du sacrifice établissent un contrat, non point entre la victime et le bourreau au courroux pervers, mais entre le martyr et celui qui voit le martyre. À ce mode violent s'oppose le sacrifice chrétien, offert à un Dieu qui refuse l'effusion sanglante. Dans Vengeances, ce type de sacrifice connaît deux modalités, selon que le coeur offert en hostie est pur ou impur: "L'un offroit un coeur doux, l'autre un coeur endurci..." (VI, v. 180). Dans son Commentaire de la Genèse, "Le Seigneur regarda à Abel et à son oblation", Calvin note que si le texte cite d'abord Abel, et si le sacrifice n'est évoqué qu'ensuite, c'est que "les œuvres n'auront nulle grâce devant Dieu, que celuy qui les a faites ne soit dejà auparavant approuvé de luy". ${ }^{14}$ Par là s'exprime une idéologie différente du sacrifice: substitution de l'acte d'offrande à l'acte d'immolation, valeur symbolique de l'oblation.

Aussi bien l'ouverture de Vengeances présente un sacrifice où l'offrande substitue le lait au sang animal: 
Ouvre ton temple saint à moy Seigneur qui veux

Ton sacré, ton secret enfumer de mes voeux, Si je n'ay or ne myrrhe à faire mon offrande Je t'apporte du lait ...

$$
\text { (v. 3-6), }
$$

et les voeux aux feux pour enfumer le saint des saints. Par là se modifient les rites, la nature de l'offrande, et la visée: “. .. et puis je porte en ton temple les dons plus agréables qu'aucun troupeau de bestes assommées: c'est un coeur abbatu, un courage atteré, une ame froissée, toutes ces parties, au pied de l'autel, comme bestes qu'on immole, ma chair esgorgée, bruslée devant toy, si bien qu'il n'en demeure que les cendres" (Médit. surles Ps. LI, p. 542). "Tu as voulu que nous te sacrifions louanges, et pour holocaustes les voeux de nos coeurs ardents; c'est ce que nous eslevons vers le ciel, c'est ce que nous deployons devant ta face" (Médit. surles Ps. LI, p. 544): dans cet échange symbolique, reste, outre la volonté de déployer un spectacle sous les yeux du Juge appelé à apprécier le "tesmoignage", l'intention de faire un pacte, d'établir, par l'offrande, un contrat qui oblige les deux parties:

Car tu ne prens plaisir au sang, l'holocauste ne plaist poinct

A toi, qui mieux aimerois l'esprit tout contrit et froissé

Point tu ne mespriseras un bon coeur submis et brisé,

Fai du bien à Sion, et rebastis son mur et ses tours:

Rassure Jerusalem, et la ceins encore de rempars.

Là l'holocauste sera tout consumé: là dis-je nos voeux

Enfumeront, comme il est enjoint, ton temple et ton autel.

(Ps. LI Vers mesurés, p. 369)

Quels que soient ses modes et ses genres, le sacrifice est la signature d'un contrat, donnant-donnant, il s'inscrit dans la structure de l'échange, du don et du contre-don, et il est à la fois réglé et régulateur. Il institue en effet un transfert: les parties offertes dans l'oblation y deviennent parties du destinataire, comme le rappelle Corneille Agrippa: "Ces sacrifices et ces oblations nous donnent . . . beaucoup de confiance, nous font de la famille de dieu, et repoussent beaucoup de maux qui nous menacent". Dans L'Hécatombe le coeur immolé devient partie du corps de Diane: "Mon cœur souffre en son coeur"(XCVI); dans Les Tragiques, chaque goutte de sang du martyr porte le nom de Dieu, et le martyr entre dans la maison et dans la famille de Dieu:

L'Eternel nous prononce et cree de sa voix

Rois, nous donnant encor plus haut nom que de Rois:

D'estrangers il nous fait ses bourgeois, sa famille,

Nous donne un nom plus doux que de fils et de fille.

(Trag. VII, v. 1073-6) 
Le sacrifice par sa violence met un terme à la violence, il institue un ordre là où régnait le désordre, il autorise le martyr à s'assurer de Dieu: rien n'oblige plus la Divinité que le sacrifice. Le contrat qu'il écrit en lettres de cendres et de sang, nulle force n'est en mesure de le rompre.

$$
* * *
$$

Ainsi s'organise dans sa remarquable cohérence l'univers imaginaire d'Aubigné, où mythes et croyances, idées et convictions, passions et fantasmes construisent un réseau obsédant où se tissent la Loi et le Lien. Comme ce glorieux Empereur, allégué dans la Meditation sur le Pseaume XVI, Aubigné peut s'écrier: "Je te salue, héritage que le Ciel me devoit!". La thématique du devoir et de la dette, du don et du contre-don, qui s'inscrit dans l'échange symbolique, marque le contrat qui lie le Prince à ses sujets, la Dame à son serviteur, la Divinité à son fidèle. Dans cette société archaïque qu'Aubigné recompose dans sa violence et son désordre, mais aussi dans son exigence de justice, dans sa demande d'équivalence, chaque peine mérite salaire, chaque bienfait doit recevoir récompense, "Et pour chaque forfait, chaque propre torment", "sang pour sang et vie pour la vie".

\section{Université de Paris VIII}

Notes

Les citations renvoient, pour Le Printemps, à l'édition procurée par IH. Weber (Paris: Presses universitaires de France, 1960), et pour tous les autres textes, à l'édition de la Pléiade (H. Weber, J. Bailbé, M. Soulié).

1 Sur la valeur symbolique du nombre cent, cf. Corneille Agrippa, La Philosophie occulte ou la Magie, trad. fr., Villain-Belhomme, 4 livres (Paris: Éd. traditionnelles, 1973-4), liv. II, chap. XV, p. 72. Jai étudié cette symbolique du nombre dans "Le nombre et la lettre. Pour une lecture du s. XCVI de L'Hécatombe", R.S.H. no 3 (1980).

2 Sur le dédoublement du destinataire, voir Starobinski, "Le style de l'autobiographie", Poétique no 3 (1970), pp. 260-1 (à propos des Confessions d'Augustin).

3 J'ai étudié les modalités du discours judiciaire dans "Structures rhétoriques des Tragiques", in La littérature de la Renaissance, Mélanges offerts à Henri Weber (Genève: Slatkine, 1984).

4 Éd. cit., pp. 385-386.

5 Voir Du Debvoir Mutuel, éd. cit., note p. 1239.

6 Ibid., p. 467.

7 Ibid., p. 478.

8 Ibid., p. 469.

9 Ibid., p. 487.

$10 \mathrm{Ibid}$., p. 489. Ici encore il faut noter la coïncidence de cette ultime séquence du traité et de l'ultime séquence du livre VII des Tragiques.

11 M. Mauss, Essai sur la nature et la fonction du sacrifice in CEuvres, 3 tomes, (Paris: éd. de Minuit, 1968-69), t. I, p. 305. 
12 Calvin distingue dans L'institution chrestienne, translatee en françois par luy mesme (Paris: $F$. Iaquy, A. Dauodeau, J. Bourgeois, 1557), chap. XVIII Dela Cene du Seigneur, p. 552, deux sortes de sacrifices, d'expiation, ou d'action de grâces.

13 Corneille Agrippa, La philosophie occulte, chap. LIX du livre troisième, "Des Sacrifices et oblations, de leurs genres et modes".

14 Calvin, Commentaires sur les cinq livres de Moyse (Genève, 1564), p. 41. 